\title{
Behandlung indolenter Non-Hodgkin-Lymphome: Neue Akzente für die Praxis
}

Symposium anlässlich der Gemeinsamen Jahrestagung der Deutschen und Österreichischen Gesellschaften für Hämatologie und Onkologie (30.9.-3.10.2001)

Mannheim, 2. Oktober 2001 
In kaum einem Bereich der Medizin gibt es so zahlreiche und vielversprechende neue Therapieansätze wie bei den indolenten Non-Hodgkin-Lymphomen (NHL). Hocheffektive Zytostatika wie Fludarabin (Fludara ${ }^{\circledR}$ ), spezifische Immuntherapeutika wie Alemtuzumab (MabCampath) oder Rituximab und mit Radionukliden beladene Antikörper wie Ibritumomab (Zevalin) stehen zur Verfügung bzw. werden in Kürze verfügbar sein.

Ziel eines Symposiums, das anlässlich der Gemeinsamen Jahrestagung der Deutschen und Österreichischen Gesellschaften für Hämatologie und Onkologie am 2. Oktober 2001 in Mannheim stattfand, war es, den Stellenwert der neuen Behandlungsverfahren für die tägliche Praxis zu definieren.

Das Chemotherapeutikum Fludarabinphosphat akkumuliert in lymphatischen Zellen, was seine hohe Wirksamkeit bei der chronisch-lymphatischen Leukämie (CLL) und anderen NHL erklärt. Neben umfassenden Daten zu Fludarabin-Mono- und Kombinationstherapien bei vorbehandelten Patienten, mit denen bereits 1996 die Zulassung für die Second-line-Therapie der CLL erwirkt wurde, liegen inzwischen auch Ergebnisse aus großen randomisierten Studien zur First-line-Therapie vor. Bei vielen Patienten können langanhaltende Remissionen erzielt werden. Damit bietet Fludarabin eine gute Grundlage für kurative Therapiekonzepte. Durch die neuen Kombinationstherapien mit Fludarabin ergeben sich faszinierende Möglichkeiten zur Eradikation von NHL bis auf die molekulare Ebene.

Patienten mit refraktärer oder rezidivierter CLL profitieren deutlich von einer Therapie mit dem humanisierten monoklonalen Antikörper CAMPATH-1H (MabCampath), der seit Juli 2001 europaweit zugelassen ist. In einer Phase-II-Studie wurde bei einem Drittel der Patienten eine Remission erzielt. Die mediane Überlebensdauer überstieg mit 16 Monaten deutlich die Erwartungen für dieses Patientenkollektiv und ist bei Respondern noch nicht erreicht.

${ }^{90}$ Yttrium-Zevalin $\left({ }^{90}\right.$ Yttrium-Ibritumomab) ist ein radioaktiv markierter monoklonaler CD20-Antikörper. Das neue Wirkprinzip der Radioimmuntherapie ermöglicht die spezifische, zielgerichtete Bestrahlung von Tumorzellen. Bei Rituximab-refraktären Patienten wurden Ansprechraten von 74\% erreicht. In einer Vergleichsstudie sprachen auf Zevalin $80 \%$ der Patienten an, auf Rituximab dagegen nur 56\% $(\mathrm{p}=0,002)$. Mit der
Zulassung für den Einsatz bei rezidivierten follikulären Lymphomen wird für das Jahr 2002 gerechnet.

\section{Therapie der chronisch-lymphatischen Leukämie}

\section{Die Primärtherapie der CLL}

Purinanaloga wie Fludarabin sind heute ein wichtiger Baustein in der Behandlung der CLL. Auch bei vorbehandelten Alkylanzien-refraktären Patienten werden damit hohe Ansprechraten erzielt. In randomisierten Studien werden derzeit sowohl der Stellenwert der Fludarabin-Monotherapie im Vergleich zur Behandlung mit Chlorambucil als auch der der Kombination Fludarabin/Cyclophosphamid im Vergleich zur FludarabinMonotherapie geprüft.

\section{Fludarabin-Monotherapie}

Die Mehrzahl der CLL-Patienten befindet sich bei Diagnosestellung im Frühstadium (Stadium Binet A). Üblicherweise werden solche Patienten nicht behandelt, sondern beobachtet. Nach dem Studienprotokoll CLL1 der Deutschen CLL-Studiengruppe wird derzeit untersucht, ob Patienten im Stadium A mit hohem Risiko für eine schnelle Progredienz von einer früh einsetzenden Fludarabin-Monotherapie profitieren. Zum Vergleich dient eine Kontrollgruppe, die nach der Randomisierung nur beobachtet wird.

Im fortgeschrittenen Stadium (Binet B und C) wird meist mit Alkylanzien (Chlorambucil, Cyclophosphamid) oder Fludarabin behandelt. Mehrere Studien, in denen Fludarabin mit Chlorambucil und Alkylanzien-haltigen Kombinationsschemata verglichen wurde, belegten deutlich höhere Ansprechraten unter Fludarabin. Dies äußerte sich auch in einem verlängerten progressions- bzw. therapiefreien Überleben (Tab. 1). Damit, so Michael Hallek, München, ist Fludarabin das potenteste Medikament, das für die Behandlung der CLL derzeit zur Verfügung steht.

Tab. 1. Studien zum Vergleich von Fludarabin mit Alkylanzien-haltigen Kombinationsschemata bei der CLL

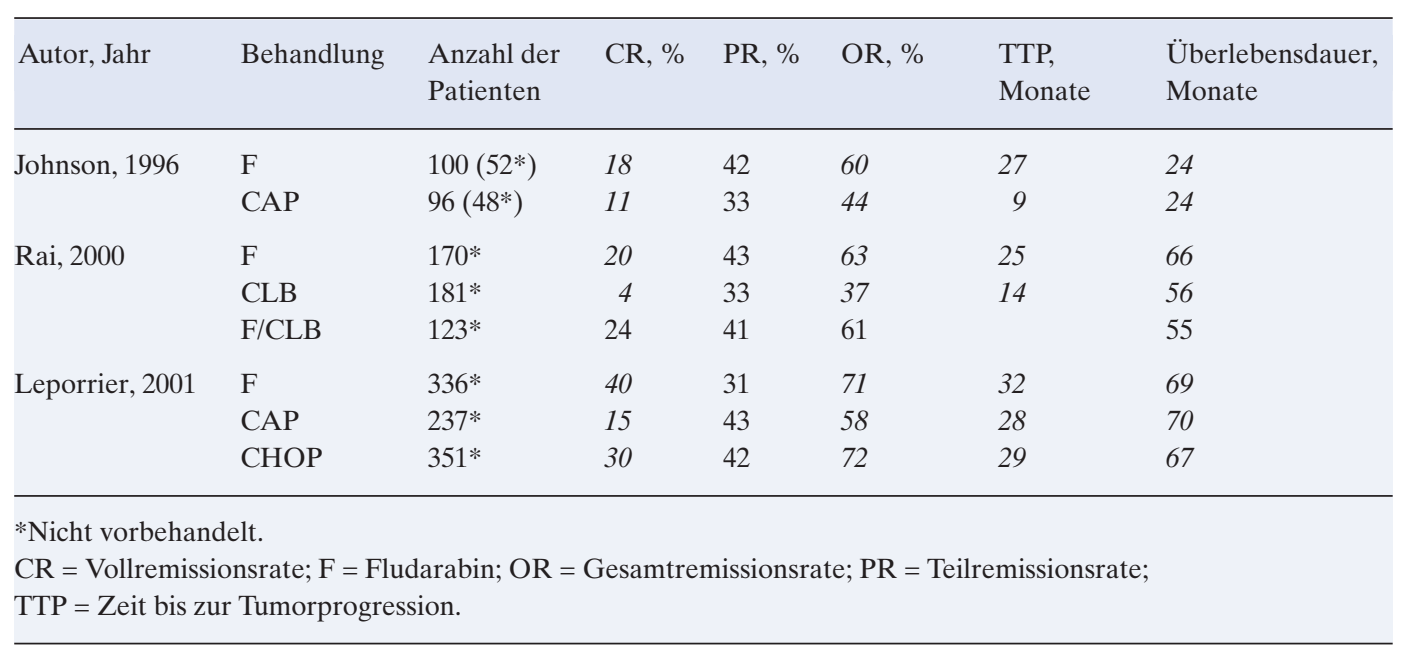


Tab. 2. Studien zur Kombination Fludarabin plus Cyclophosphamid

\begin{tabular}{|c|c|c|c|c|c|}
\hline Autor, Jahr & Schema & $\begin{array}{l}\text { Anzahl der } \\
\text { Patienten }\end{array}$ & $\mathrm{CR}, \%$ & $\mathrm{PR}, \%$ & OR, \% \\
\hline O’Brien, 2001 & F $30 \mathrm{mg} / \mathrm{m}^{2} \&$ C $500 / 350 / 300 \mathrm{mg} / \mathrm{m}^{2}$ Tag $1-3$ & 34 CLL* $^{*}$ & 35 & 53 & 88 \\
\hline Hallek, 2001 & $\mathrm{~F} 25 \mathrm{mg} / \mathrm{m}^{2} \& \mathrm{C} 250 \mathrm{mg} / \mathrm{m}^{2}$ Tag $1-3$ & 36 CLL & 16 & 75 & 91 \\
\hline \multirow[t]{2}{*}{ Frewin, 1999} & F $25 \mathrm{mg} / \mathrm{m}^{2} \&$ C $250 \mathrm{mg} / \mathrm{m}^{2}$ Tag $1-3$ & $10 \mathrm{NHL}$ & 0 & 50 & 50 \\
\hline & & 7 CLL & 28 & 43 & 71 \\
\hline \multirow[t]{2}{*}{ Flinn, 2000} & F $20 \mathrm{mg} / \mathrm{m}^{2}$ Tag $1-5$ \& C $600 \mathrm{mg} / \mathrm{m}^{2}$ Tag 1 , G-CSF $5 \mu \mathrm{g} / \mathrm{kg}$ Tag $8+$ & $43 \mathrm{NHL}$ & 60 & 32 & 92 \\
\hline & & $17 \mathrm{CLL}$ & 47 & 53 & 100 \\
\hline
\end{tabular}

\section{Fludarabin plus Cyclophosphamid}

Um die Behandlungsergebnisse bei der fortgeschrittenen CLL weiter zu verbessern, werden vier verschiedene Konzepte geprüft: die Kombination von Fludarabin mit anderen Zytostatika, die Gabe monoklonaler Antikörper, die Hochdosis-Therapie und der Einsatz neuer Medikamente. Besonders wirksam scheint hier die Kombination aus Fludarabin und Cyclophosphamid (FC) zu sein, für welche in In-vitro-Untersuchungen ein synergistischer Effekt gezeigt werden konnte. In vier kürzlich publizierten klinischen Studien zur FC-Kombination bei größtenteils vorbehandelten Patienten wurden Gesamtansprechraten von etwa 90\% erzielt (Tab. 2). Damit, so Hallek, gehört die FC-Kombination zu den vielversprechendsten Therapieoptionen. Selbst Patienten, die auf eine Cyclophosphamid- oder Fludarabin-Monotherapie nicht mehr ansprechen, profitieren von dieser Kombination.

\section{Monoklonaler Antikörper CAMPATH-1H}

Mit dem monoklonalen Antikörper CAMPATH-1H (Alemtuzumab) wurden bei vorbehandelten Fludarabin-refraktären BCLL-Patienten Gesamtansprechraten von zirka 33\% erzielt. Dies ist das Ergebnis der zulassungsrelevanten Studie [Keating et al., 1999] und weiterer unterstützender Phase-II-Studien [Rai et al., 2000b; Kennedy et al., 1999; Österborg et al., 1997]. Im peripheren Blut konnten die Leukämiezellen in diesen Studien fast vollständig (97\%), im Knochenmark zu über 60\% durch CAMPATH-1H reduziert werden. Bei der First-line-Therapie mit CAMPATH-1H fielen die hohen Ansprechraten von annähernd $100 \%$ im peripheren Blut und von über $80 \%$ im Knochenmark auf. CAMPATH-1H ist somit eine ideale Substanz, um die minimale Resterkrankung zu eliminieren, betonte Hallek.

\section{Phase-III-Studie der Deutschen CLL-Studiengruppe mit CAMPATH-1H}

Vor diesem Hintergrund werden in dem Studienprotokoll CLL4 der Deutschen CLL-Studiengruppe (DCLLSG) nicht vorbehandelte, maximal 65-jährige Patienten im Stadium Binet $\mathrm{B} / \mathrm{C}$ zunächst entweder mit 6 Zyklen Fludarabin oder mit 6 Zyklen FC behandelt. Patienten beider Therapiearme, die eine Voll- oder Teilremission zeigen, erhalten im CLL4B-Protokoll anschließend CAMPATH-1H als Konsolidierungstherapie oder werden beobachtet (Abb. 1).

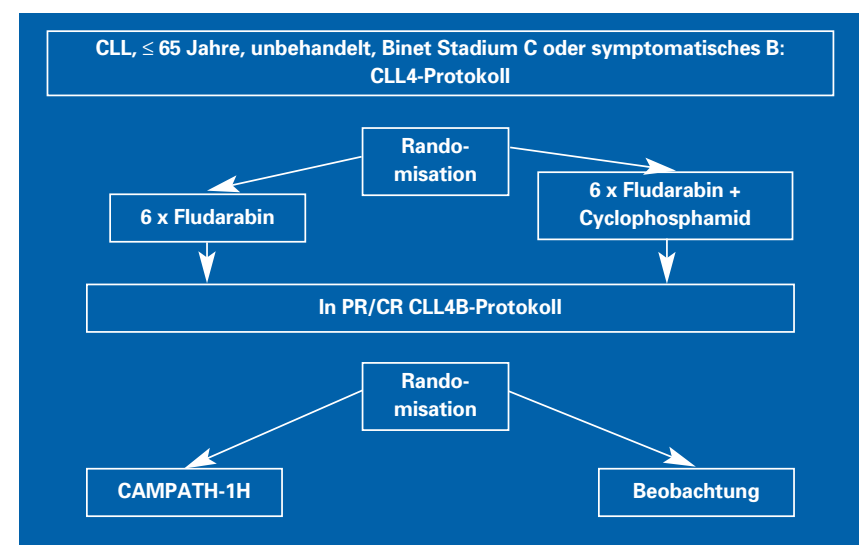

Abb. 1. Phase-III-Studie der Deutschen CLL-Studiengruppe: Fludarabin versus Fludarabin plus Cyclophosphamid gefolgt von CAMPATH$1 \mathrm{H}$ zur Remissionserhaltung.

Wie eine erste Zwischenauswertung zeigt, ist die FludarabinMonotherapie mit der FC-Kombination hinsichtlich der Toxizität vergleichbar. Die Ansprechrate für beide Arme liegt derzeit zusammengefasst bei $90 \%$, eine getrennte Analyse ist noch nicht möglich.

In Kürze, so Hallek, wird Fludarabin als erstes Purinanalogon auch oral verfügbar sein. Effektivität und Verträglichkeit sind mit intravenösem Fludarabin vergleichbar, die Therapie wird aber für die Patienten deutlich erleichtert.

\section{Die Therapie der rezidivierenden und refraktären CLL}

Patienten mit rezidivierter und Therapie-refraktärer CLL haben Grund zum Optimismus, so Michael J. Keating, Houston, TX, USA, denn die Therapieoptionen haben sich erheblich verbessert, und weitere neue Präparate befinden sich in der Entwicklung. Besonders viel versprechend ist der monoklonale Antikörper CAMPATH-1H, der seit August 2001 in Deutschland verfügbar ist. 
Auf Fludarabin sprechen etwa die Hälfte der Patienten mit rezidivierter und Alkylanzien-refraktärer CLL an, bei refraktären Patienten mit Alkylanzien-sensitiver CLL fast alle (96\%). Inzwischen, so Keating, gibt es jedoch zahlreiche Patienten, die nicht nur Alkylanzien-refraktär, sondern auch Fludarabin-refraktär sind, und aufgrund dessen eine geringere Lebenserwartung haben. Gibt man diesen Patienten Fludarabin in Kombination z.B. mit Cyclophosphamid, kann das Überleben deutlich verlängert werden.

Als weitere Therapieoption steht der monoklonale Anti-CD20Antikörper Rituximab zur Verfügung, der bei CLL aber nur in sehr hoher Dosis oder bei dosisintensivierter Anwendung (dreimal wöchentlich) wirksam ist. Auch die Dreifachkombination Fludarabin/Cyclophosphamid/Rituximab wurde geprüft. Die Ansprechrate bei mit Fludarabin vorbehandelten Patienten liegt bei $66 \%$. Allerdings steigert Rituximab auch die Toxizität der Chemotherapie: Wegen der häufig auftretenden Tumorlyse müssen zudem alle Patienten zusätzlich Allopurinol erhalten.

\section{CAMPATH-1H - die Zulassungsstudie}

Der neue Anti-CD52-Antikörper CAMPATH-1H (Wirkstoff Alemtuzumab, Handelsname MabCampath) hat sich bei $\mathrm{Pa}-$ tienten mit CLL als sehr wirksam erwiesen. In der Zulassungsstudie, die bei Patienten mit Alkylanzien-vorbehandelter, Fludarabin-refraktärer CLL durchgeführt wurde, konnte unter der Monotherapie mit CAMPATH-1H eine Ansprechrate von 33\% erzielt werden. In der historischen Vergleichsgruppe des M.D. Anderson Cancer Center (MDACC) in Houston war es unter einer herkömmlichen Chemotherapie nur bei 23\% der Patienten zu einer Remission gekommen. Die Überlebenszeit zeigte mit median 16 versus 9 Monaten ebenfalls einen deutlichen Unterschied (Tab. 3). Dabei war die mediane Überlebensdauer bei den «Respondern» signifikant länger als die des Gesamtkollektivs und wurde bis heute noch nicht erreicht (Abb. 2). Auch hinsichtlich der Frühtodesfälle schnitt die Immuntherapie günstiger ab: 5\% gegenüber 14\% im historischen Kontrollkollektiv. Eine Aufschlüsselung der Ansprechraten in Abhängigkeit von verschiedenen Prognosefaktoren macht zweierlei deutlich: Zum einen wird offensichtlich, dass es sich um Patienten in einem sehr weit fortgeschrittenen Krankheitsstadium handelt. Zum anderen ist erkennbar, dass CAMPATH-1H selbst bei diesen Patienten mit schlechtester Prognose noch eine vielversprechende Wirkung zeigt.

Besonders spektakulär, so Keating, ist die hohe Wirksamkeit von CAMPATH-1H, Leukämiezellen im peripheren Blut sowie in Knochenmark und Milz zu eliminieren. CAMPATH-1H wurde aufgrund dieser Eigenschaften auch schon zur Behandlung der minimalen Restkrankheit nach Chemotherapie eingesetzt. Bei etwa 50\% der Patienten konnte dadurch PCR-Negativität erreicht werden.

In der Zulassungsstudie verbesserte sich nach der CAMPATH1H-Therapie die klinische Symptomatik sogar bei den «NonRespondern». Beispielsweise kam es bei 73\% der «Responder» und bei 39\% der «Non-Responder» zu einem Anstieg des HbWerts um mindestens $2 \mathrm{~g} / \mathrm{dl}$. B-Symptome bzw. Fatigue gingen bei $100 \%$ der «Responder» und bei $67 \%$ der «Non-Responder» zurück. Dies bedeutet, so Keating, dass selbst bei den «NonRespondern» eine eindeutige Verbesserung der Lebensqualität erzielt werden konnte.
Tab. 3. Monotherapie mit CAMPATH-1H bei Fludarabin-refraktären CLL-Patienten

\begin{tabular}{|c|c|c|}
\hline Ergebnis & $\begin{array}{l}\text { CAMPATH-1H } \\
(\mathrm{n}=93)\end{array}$ & $\begin{array}{l}\text { Vergleichsgruppe } \\
(\mathrm{n}=147)\end{array}$ \\
\hline $\mathrm{CR}, \%$ & 2 & 2 \\
\hline $\mathrm{PR}, \%$ & $31\} 33$ & $21\} 23$ \\
\hline $\mathrm{ED}, \%$ & 5 & 14 \\
\hline $\begin{array}{l}\text { Mediane Überlebensdauer, } \\
\text { Monate }\end{array}$ & 16 & 9 \\
\hline
\end{tabular}

$\mathrm{ED}=$ Frühtodesrate

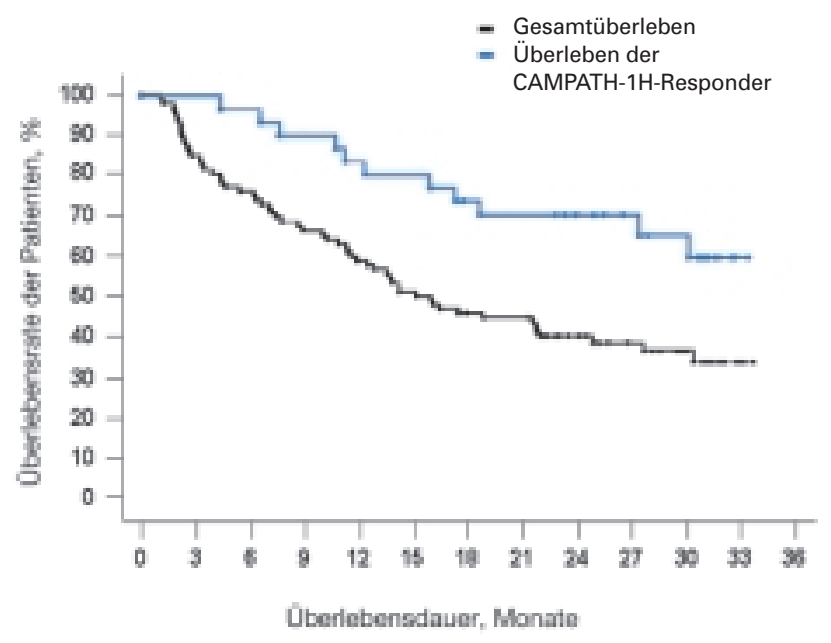

Abb. 2. CAMPATH-1H-Zulassungsstudie: Medianes Überleben der «Responder» und Gesamtüberleben

\section{Therapie follikulärer Non-Hodgkin-Lymphome}

\section{Strategien für die Primär- und Rezidivtherapie}

Das follikuläre Lymphom stellt mit etwa 25\% einen der häufigsten histologischen Subtypen der NHL dar. Das Gesamtüberleben der Patienten in den Stadien III und IV wird durch eine konventionelle Chemotherapie nicht beeinflusst. Seit einiger Zeit stehen jedoch neue, innovative Therapieansätze zur Verfügung.

Eine Interferon- $\alpha$-Erhaltungstherapie nach einer zytoreduktiven Chemotherapie führte in mehreren Studien zu einem verlängerten progressionsfreien Überleben. In einer prospektiv randomisierten, multizentrischen Studie der Deutschen Studiengruppe für niedrigmaligne Lymphome (GLSG) wurde eine derartige Erhaltungstherapie mit einer konsolidierenden Hochdosis-Therapie und autologer Stammzelltransplantation verglichen. In dem Behandlungsarm mit Hochdosis-Therapie konnte die Rezidivrate im Vergleich zur Interferon- $\alpha$-Erhaltungstherapie nahezu halbiert werden. Aufgrund der bekannten Spättoxizitäten der Hochdosis-Therapie, speziell sekundärer Neoplasien, steht eine abschließende Beurteilung des Gesamtüberlebens allerdings noch aus. 


\section{Neuer Standard für die Rezidivtherapie}

Die bisher höchsten publizierten Remissionsraten bei Patienten mit indolenten Lymphomen werden durch Fludarabin-haltige Polychemotherapien erzielt. Dies ist in mehreren Phase-II- und Phase-III-Studien belegt, sagte Martin Dreyling, München.

In einer prospektiv randomisierten Studie der GLSG wurde jetzt der zusätzliche Effekt der Gabe des monoklonalen Anti-CD20Antikörpers Rituximab evaluiert. Alle Patienten litten an einem rezidivierten, indolenten Lymphom (zwei Drittel follikuläre Lymphome, ein Drittel Mantelzell-Lymphome). Sie erhielten 4 Zyklen der Dreifachkombination FCM (Fludarabin, Cyclophosphamid, Mitoxantron). Bei der Hälfte der Patienten wurde nach erfolgter Randomisierung zusätzlich Rituximab gegeben. Das Therapieregime ist in der Tabelle 4 zusammengestellt.

Von 80 der insgesamt 147 Patienten liegen erste Ergebnisse vor. Wie die Toxizitätsdaten zeigen, war die Vierfachkombination mit Rituximab gut verträglich und unterschied sich diesbezüglich kaum von der Dreifachkombination. Die Remissionsrate unter FCM plus Rituximab war jedoch schon in dieser Zwischenauswertung statistisch signifikant höher als unter FCM alleine (89\% versus 53\%; $\mathrm{p}=0$,000715) (Tab. 5). Der Prozentsatz der Vollremissionen unter FCM plus Rituximab war sogar mehr als doppelt so hoch (36\% versus $15 \%$ ). Aus diesem Grunde wurde die Randomisation zu dieser Fragestellung beendet und FCM plus Rituximab als neuer Standard für die Rezidivtherapie bezeichnet.

Tab. 4. FCM versus FCM plus Rituximab - das Therapieregime

\begin{tabular}{lrl}
\hline & Dosis, $\mathrm{mg} / \mathrm{m}^{2}$ & Tag \\
\hline FCM & & \\
$\quad$ Fludarabin & 25 & $1-3$ \\
Cyclophosphamid & 200 & $1-3$ \\
Mitoxantron & 8 & 1 \\
& & \\
FCM plus Rituximab & & \\
Fludarabin & 25 & $1-3$ \\
Cyclophosphamid & 200 & $1-3$ \\
Mitoxantron & 8 & 1 \\
Rituximab & 375 & 0 \\
\hline
\end{tabular}

Tab. 5. FCM versus FCM plus Rituximab - die ersten Ergebnisse

\begin{tabular}{|c|c|c|c|c|}
\hline & FCM & & $\mathrm{FCM}+$ & uximab \\
\hline & $\mathrm{n}$ & $\%$ & $\mathrm{n}$ & $\%$ \\
\hline Auswertbare & & & & \\
\hline Patienten: & 39 & & 41 & \\
\hline Chemotherapie & & & & \\
\hline vollständig: & 34 & & 36 & \\
\hline $\mathrm{CR}$ & & 15 & 13 & 36 \\
\hline PR & $13\} 18$ & $38\} 53$ & $19\} 32$ & $53\} 89$ \\
\hline $\mathrm{SD} / \mathrm{MR}$ & 2 & 6 & 0 & 0 \\
\hline PD & 11 & 32 & 3 & 8 \\
\hline ED & 3 & 9 & 1 & 3 \\
\hline
\end{tabular}

$\mathrm{SD}=$ Stabile Erkrankung; $\mathrm{MR}=$ minimale Remission $; \mathrm{PD}=$ Progression des Lymphoms.

\section{Radioimmuntherapie - eine neue Option zur Rezidiv- therapie indolenter Non-Hodgkin-Lymphome}

Die Entwicklung der zielgerichteten Immuntherapie war ein großer Fortschritt in der Therapie indolenter B-Zell-NHL. Denn obgleich mit dem Anti-CD20-Antikörper Rituximab hohe Remissionsraten erzielt werden, ist diese Behandlung nicht kurativ. Sehr vielversprechend ist hier der Einsatz radioaktiv markierter Antikörper.

Die bislang umfassendste klinische Erfahrung liegt mit ${ }^{131} \mathrm{Jod}-$ bzw. ${ }^{90}$ Yttrium-markierten Anti-CD20-Antikörpern vor. Gegenüber ${ }^{131}$ Jod-markierten Antikörpern hat der ${ }^{90}$ Yttrium-markierte Antikörper Ibritumomab (Zevalin) den Vorteil, dass ${ }^{90}$ Yttrium keine weitreichende Gammastrahlung aussendet. Anders als bei der ${ }^{131}$ Jod-Markierung werden entfernte Organe somit nicht bestrahlt, und maligne Tumorzellen sind zudem einer höheren, zielgenauen Betastrahlung ausgesetzt. Darüber hinaus ist die Halbwertszeit von ${ }^{90}$ Yttrium wesentlich kürzer, und die Patienten müssen nicht, wie bei einer ${ }^{131}$ Jod-Therapie, aus Strahlenschutz-Gründen über lange Zeiträume in spezielle nuklearmedizinische Einrichtungen eingewiesen werden, sondern lassen sich prinzipiell sogar ambulant behandeln, sagte Martin Bentz, Ulm.

\section{${ }^{90}$ Yttrium-Ibritumomab $\left({ }^{90}\right.$ Yttrium-Zevalin) versus Rituximab}

${ }^{90}$ Yttrium-Ibritumomab wurde bereits in mehreren klinischen Studien geprüft. In einer von Witzig et al. [2000a] durchgeführten Phase-III-Studie bei 143 Patienten mit rezidiviertem oder primär refraktären, CD20-positiven, indolenten oder transformierten NHL wurde die Wirksamkeit von Zevalin mit der von Rituximab verglichen. Die Patienten erhielten randomisiert entweder Rituximab (375 mg/m² wöchentlich über 4 Wochen) oder eine einwöchige Radioimmuntherapie mit Rituximab in reduzierter Dosis und ${ }^{90}$ Yttrium-Ibritumomab. Die Vorweggabe von Rituximab wurde gewählt, um die CD20-Bindungsstellen im peripheren Blut zu besetzen und so die Radioimmuntherapie mit ${ }^{90}$ Yttrium-Ibritumomab noch zielgerichteter einsetzen zu können. Das Ergebnis war hochsignifikant zugunsten der Radioimmuntherapie mit einer Vollremissionsrate von $30 \%$ versus $16 \%(p=0,04)$ und einer Gesamtansprechrate von $80 \%$ versus $56 \%(p=0,002)$ (Tab. 6).

In einer zweiten Studie bei 54 Patienten mit Rituximab-refraktärem follikulären NHL konnte mit ${ }^{90}$ Yttrium-Ibritumomab eine Gesamtansprechrate von $74 \%$ erreicht werden, darunter $15 \%$ Vollremissionen [Witzig et al., 2000b].

Eine Subanalyse, die 71 über 65-jährige Patienten aus vier Studien mit ${ }^{90}$ Yttrium-Ibritumomab umfasste, konnte bei diesem Kollektiv eine Gesamtansprechrate von $68-76 \%$ belegen, und die Verträglichkeit war vergleichbar mit der jüngerer Patienten [Emmanoulides et al., 2001].

Mit der Zulassung von Zevalin in den USA wird bereits für Ende 2001 gerechnet. In Europa werden derzeit zwei große klinische Studien durchgeführt: Zum einen wird bei Patienten mit follikulärem Lymphom, die mittels Primärtherapie eine Volloder Teilremission erreicht haben, Zevalin als Remissionserhaltungstherapie untersucht. Zum anderen wird Zevalin bei Patienten mit rezidivierten oder primär refraktären diffus-großzelligen NHL geprüft. 
Tab. 6. Phase-III-Studie mit Zevalin versus Rituximab bei rezidivierten oder primär refraktären NHL

\begin{tabular}{llll}
\hline & $\begin{array}{l}\text { Zevalin } \\
(\mathrm{n}=73)\end{array}$ & $\begin{array}{l}\text { Rituximab } \\
(\mathrm{n}=70)\end{array}$ & $\mathrm{p}$-Wert \\
\hline OR, \% & 80 & 56 & 0,002 \\
Follikuläres NHL & 86 & 55 & \\
CR & 30 & 16 & 0,040 \\
CRu & 4 & 4 & \\
PR & 45 & 36 & \\
\hline
\end{tabular}

$\mathrm{CRu}=$ Unbestätigte komplette Remission.

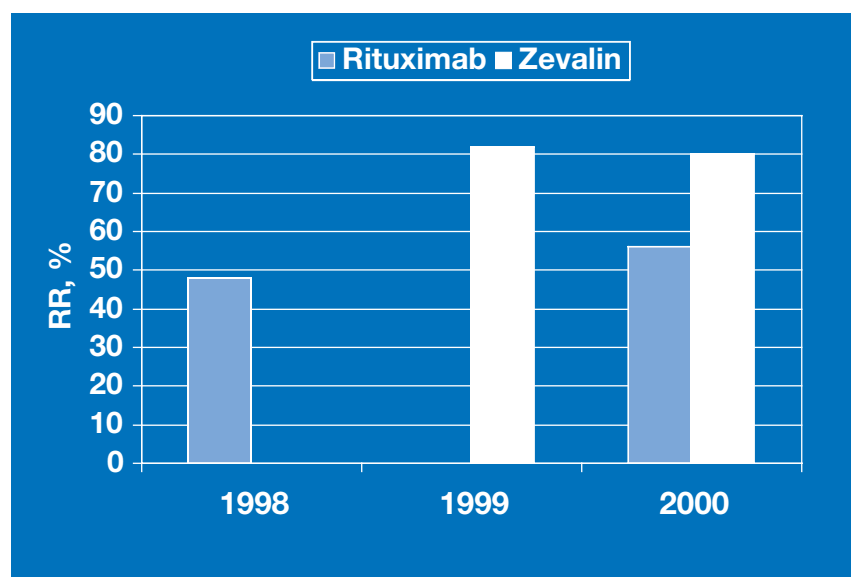

Abb. 3. Immuntherapie rezidivierter indolenter Lymphome: Fortschritt durch die Radioimmuntherapie.

1998: Phase II Rituximab: 48\% RR [McLaughlin et al., 1998].

1999: Phase I/II Zevalin: 82\% RR [Witzig et al., 1999].

2000: Phase III Zevalin versus Rituximab: $80 \%$ versus 56\% RR, $\mathrm{p}=0,002$ [Witzig et al., 2000a].

Vergleicht man die mit Rituximab erzielbaren Remissionsraten mit den in den Jahren 1999 und 2000 mit Zevalin erreichten Ergebnissen, wird deutlich, so Bentz, dass von Zevalin definitiv ein zusätzlicher Effekt zu erwarten ist (Abb. 3).

\section{Diagnostik}

\section{Perspektiven der molekularen Diagnostik für die Therapieplanung bei indolenten Lymphomen}

Die Kenntnis genetischer Veränderungen in Lymphomzellen hat sowohl für die Identifizierung pathogenetisch relevanter Gene als auch für die Einteilung von NHL-Subgruppen große Bedeutung erlangt. Die möglichen Implikationen der molekularen Diagnostik für Prognose und Therapie der NHL/CLL sind enorm.

Techniken, die im Rahmen der molekularen Diagnostik zur Anwendung kommen, sind z.B. die Polymerase-Kettenreaktion (PCR) und die DNA-Sequenzanalyse, die unter anderem zur Bestimmung des Mutationsstatus der variablen Immunglobulin-Gene ( $\mathrm{IgV}$ ) bei der CLL eingesetzt werden können, sowie die Fluoreszenz-in-situ-Hybridisierung (FISH), die komparati- ve genomische Hybridisierung ( $\mathrm{CGH}$ ) und die DNA-ChipTechnologie. Mittels FISH und DNA-Chip-Technologie lassen sich z.B. bei der CLL genomische Aberrationen nachweisen und spezifische genetische Veränderungen identifizieren, die eine hohe prognostische Aussagekraft besitzen. Durch die CGH-Analyse und die DNA-Chip-Technologie kann möglicherweise bei den nodalen Lymphomen ein ähnlicher Fortschritt erzielt werden.

Aufgrund der genannten Diagnostik weiß man heute, so Hartmut Döhner, Ulm, dass bei der CLL zwei verschiedene Varianten derselben Erkrankung existieren: eine Variante ohne somatische IgV-Mutationen, die sich aus B-Zell-Progenitor-Zellen entwickelt, und eine andere Variante mit somatischen IgV-Mutationen, die aus Post-Keimzentrumszellen stammt. Die Prognose der IgV-mutierten CLL ist wesentlich besser als die der nichtmutierten, d.h. die Gesamtüberlebensdauer der Patienten ist erheblich länger.

Auch die Art der genomischen Aberration hat einen Einfluss auf das Überleben. So haben z.B. Patienten mit einer 17p-Deletion, welche das die Apoptose regulierende p53-Gen umfasst, die schlechteste Prognose, hingegen Patienten mit einer 13qDeletion die beste.

Wie multivariate Analysen gezeigt haben, ist die prognostische Aussagekraft des IgV-Mutationsstatus und der Existenz einer 17p-Deletion wesentlich größer als die des Krankheitsstadiums nach Binet, des Alters oder der Leukozytenzahl. Für die künftige Therapieplanung sollte der molekularen Diagnostik deshalb ein hoher Stellenwert zukommen.

\section{Literatur}

Emmanoulides C, et al: Proc ASCO 2001;20:286a (Abstract 1143).

Johnson S, et al: Lancet 1996;347:1432-1438.

Flinn IW, et al: Blood 2000;96:71-75.

Frewin R, et al: Br. J Haematol 1999;104:612-613.

Hallek M, et al: Br J Haematol 2001;114:342-348.

Keating MJ, et al: Blood 1999:94,10:705a(Abstract 3118).

Kennedy B, et al: Blood 1999;94,10:603a(Abstract 2683).

Leporrier M, et al: Blood 2001;98:2319-2325.

Mc Laughlin P, et al: J Clin Oncol 1998;16:2825-2833.

O'Brien SM, et al: J Clin Oncol 2001;19:1414-1420.

Österborg A, et al: J Clin Oncol 1997;15:1567-1574.

Rai K, et al: Blood 2000b;96,11:163a(Abstract 703).

Rai K, et al: N Engl J Med 2000;343:1750-1757.

Witzig TE, et al.: J Clin Oncol 1999;17:3793-3803.

Witzig TE, et al: Blood 2000;96,11:831a(Abstract 3591).

Witzig TE, et al: Blood 2000b;96,11:507a(Abstract 2183).

\section{Impressum}

Beilage zu Onkologie, Band 24, Heft 6, Dezember 2001

Mit freundlicher Unterstützung durch MedacSchering Onkologie GmbH, München, und Schering Wien Ges.m.b.H,

Die Wiedergabe von Gebrauchsnamen, Handelsnamen, Warenbezeichnungen etc. in dieser Beilage auch ohne besondere Kennzeichnung berechtigen nicht zu der Annahme, dass solche Namen im Sinne der Warenzeichen- und Markenschutz-Gesetzgebung als frei zu betrachten wären und daher von jedermann benutz werden dürften. Für Angaben über Dosierungsanweisungen und Applikationsformen kann vom Verlag keine Gewähr übernommen werden. Derartige Angaben müssen vom jeweiligen Anwender im Einzelfall anhand anderer Literaturstellen auf ihre Richtigkeit überprüft werden.

Alle Rechte vorbehalten.

Ohne schriftliche Genehmigung des Verlags dürfen diese Publikation oder Teile daraus nicht in andere Sprachen übersetzt oder in irgendeiner Form mit mechanischen oder elektronischen Mitteln (einschließlich Fotokopie, Tonaufnahme und Mikrokopie) reproduziert oder auf einem Datenträger oder Computersystem gespeichert werden.

(c) Copyright 2001 by S. Karger GmbH, Postfach, D-79095 Freiburg, und S. Karger AG, Postfach, CH-4009 Basel

Druck: KONKORDIA GmbH, Bühl - Das Medienunternehmen 